\title{
Overlaps with arbitrary two-site states in the XXZ spin chain
}

\author{
B. Pozsgay ${ }^{1,2}$ \\ ${ }^{1}$ Department of Theoretical Physics, Budapest University \\ of Technology and Economics, 1111 Budapest, Budafoki út 8, Hungary \\ ${ }^{2}$ BME Statistical Field Theory Research Group, Institute of Physics, \\ Budapest University of Technology and Economics, H-1111 Budapest, Hungary
}

January 26, 2018

\begin{abstract}
We present a conjectured exact formula for overlaps between the Bethe states of the spin-1/2 XXZ chain and generic two-site states. The result takes the same form as in the previously known cases: it involves the same ratio of two Gaudin-like determinants, and a product of single-particle overlap functions, which can be fixed using a combination of the Quench Action and Quantum Transfer Matrix methods. Our conjecture is confirmed by numerical data from exact diagonalization. For one-site states the formula is found to be correct even in chains with odd length, where existing methods can not be applied. It is also pointed out, that the ratio of the Gaudin-like determinants plays a crucial role in the overlap sum rule: it guarantees that in the thermodynamic limit there remains no $\mathcal{O}(1)$ piece in the Quench Action.
\end{abstract}

\section{Introduction}

Integrable quantum mechanical models are special many-body theories, where the eigenvalues and eigenstates of the Hamiltonian are known exactly 伍, 22. Moreover, in many cases even thermodynamical properties and correlation functions can be computed [3, 国, 可. There is a vast literature devoted to the study of equilibrium properties of models such as the XXX and XXZ chains, which play a central role due to their relative simplicity despite their interacting nature, and their experimental relevance. Recently an increasing attention has been focused on the study of overlaps. The main question is: how can we compute the overlaps between exact eigenstates of integrable models and certain "initial states", and what is the class of states for which exact analytic results are to be expected. The motivation to study this problem comes from (at least) three different areas of research: Quantum Quenches, the AdS/CFT conjecture, and finite temperature problems.

First of all, the study of non-equilibrium situations raised the question whether isolated integrable systems equilibrate to Generalized Gibbs Ensembles (GGE's) [6, 7, 8]. One way towards a definite answer is to compute the long time limit of observables from first principles and to compare it to the prediction of the GGE. This approach relies on the knowledge of the overlaps: they are used as an input for the Quench Action (QA) method [9], which selects those Bethe states that are relevant for the long-time limit of local observables. Thus it was necessary to obtain exact formulas for the overlaps with Bethe states, and this program has been carried out for certain initial states of the spin- $1 / 2$ XXZ model [10, 11, 12, 13. These results lead to the understanding that the GGE built from the so-called ultra-local charges is not sufficient to describe the stationary states [14, 15, but an extended GGE that incorporates all the (recently discovered) quasi-local charges $\mid 16$ gives correct predictions [17], at least for the XXZ chain. 
The Complete GGE specified in 17 (see also $[18,19,20]$ ) is self-sufficient without the knowledge of the overlaps: it completely determines the relevant Bethe states through certain relations called the "string-charge duality" 21]. The effectiveness of this approach has been demonstrated for different types of initial states in [17, 22, 20]. This raises the question whether the study of overlaps is still relevant for the non-equilibrium problems. We believe the answer is a definite yes. On the one hand, the overlaps are necessary ingredients for certain methods computing the finite time dynamics [23, 24, 25]. On the other hand, in systems with higher rank symmetries the overlaps "lead the way" once again: there are cases where the QA method is worked out [26] using exact overlap formulas [27], but the GGE is not yet established. Also, a different viewpoint was laid out in [28, which focused on the question of which initial states can be considered "integrable", i.e. when do common features of integrability show up in the overlaps, the time evolution, and the stationary states. It was argued in 28] that very generally it is the subclass of integrable initial states where exact (factorized) overlap formulas can be expected. Having a precise definition of integrability and different methods to test it, it is now an interesting problem to find exact overlap formulas for integrable initial states, beyond the known cases, both in the spin-1/2 XXZ chain and other models. Closely related questions about overlaps have been investigated in Integrable QFT's in the papers [29, 30, 31].

A second motivation to study exact overlap formulas comes from the AdS/CFT conjecture, where the overlaps describe one-point functions of composite operators [32, 33. This line of research led to new overlap formulas, which include overlaps with integrable MPS's in the spin-1/2 XXX chain [32, 34, 33 and the $S U(3)$-symmetric model too 227. In the XXX chain the MPS's in question were shown to be zero-momentum components of two-site states or finitely entangled states obtained by the action of transfer matrices on two-site states, whereas the interpretation of the MPS of [27] within integrability is not yet known.

Finally, the third motivation to study overlaps comes from finite temperature problems: It was shown in 35] that the boundary free energy of the XXZ spin chain can be calculated in the Quantum Transfer Matrix (QTM) framework by the Trotter limit of an exact overlap (see also $[36 \mid$ ). The situation is analogous to integrable boundary QFT, where the object in question is the exact overlap between the finite volume ground state and a boundary state [37, 38], although in QFT this quantity is computed more easily from Thermodynamic Bethe Ansatz [39, 40].

Regarding the XXZ spin chain, existing overlap formulas [10, 11, 12, 13, 34 concern two-site states that are described by the diagonal $K$-matrices (see main text for definitions). The most studied examples are the Néel and dimer states. On the other hand, there are exact results available for quenches from other two-site states [41], although these results apply to the thermodynamic limit directly. It was shown in 28 that in the spin- $1 / 2$ case all two-site states are integrable, therefore it is natural to expect that there are exact finite volume overlap formulas for arbitrary two-site states. The construction of such formulas is the problem that we investigate in the present work.

In the next section we collect a number of existing results for overlaps and quenches, and we point out an important link between the relevant finite volume and infinite volume calculations. This connection is then used in Section 3 to conjecture an exact overlap formula for generic $K$-matrices. At present we do not have a proof of our result; numerical checks confirm its validity, and it is shown to have the correct thermodynamic limit. In Subsection 2.5 we also point out an interesting relation between the Gaudin-like determinants appearing in the overlaps and the calculation of the overlap sum rule within the Quench Action framework. 


\section{Overlap formulas - Ingredients}

\subsection{The model and its Bethe Ansatz solution}

We consider the anti-ferromagnetic spin-1/2 XXZ Heisenberg model on a chain of length $L$ with periodic boundary conditions. The Hamiltonian is

$$
H=\sum_{j=1}^{L}\left(\sigma_{j}^{x} \sigma_{j+1}^{x}+\sigma_{j}^{y} \sigma_{j+1}^{y}+\Delta\left(\sigma_{j}^{z} \sigma_{j+1}^{z}-1\right)\right) .
$$

Here $\Delta$ is the anisotropy parameter. In this work we will focus on the massive regime $(\Delta>1)$, but we expect that our results will be applicable to most states even in the $\Delta<1$ case 1 .

This Hamiltonian can be diagonalized by the Bethe Ansatz [43, 44, 45, 46]. The eigenstates are constructed as interacting spin waves over a ferromagnetic reference state and they are characterized by a set of rapidities $\{\lambda\}_{N}$; for the states we will use the notation $\left|\{\lambda\}_{N}\right\rangle$. In the coordinate Bethe Ansatz representation the un-normalized wave function can be written as

$$
\Psi_{L}\left(\lambda_{1}, \ldots, \lambda_{N} \mid s_{1}, \ldots, s_{N}\right)=\sum_{P \in \sigma_{N}} \prod_{j} F\left(\lambda_{P_{j}}, s_{j}\right) \prod_{j>k} \frac{\sin \left(\lambda_{P_{j}}-\lambda_{P_{k}}-i \eta\right)}{\sin \left(\lambda_{P_{j}}-\lambda_{P_{k}}\right)}
$$

with

$$
F(\lambda, s)=\sinh (\eta) \sin ^{s-1}(\lambda+i \eta / 2) \sin ^{L-s}(\lambda-i \eta / 2) .
$$

Here $s_{j}$ denote the positions of the down spins over a ferromagnetic reference state with all spins up, and we assume $s_{j}<s_{k}$ for $j<k$. The parameter $\eta$ is given by the relation $\Delta=\cosh \eta$ and the rapidities $\left\{\lambda_{j}\right\}$ characterize the spin waves. The Bethe wave function is invariant with respect to a shift $\lambda_{j} \rightarrow \lambda_{j}+\pi$ for every $j$ (up to an irrelevant phase for odd $L)$, therefore we assume $\Re\left(\lambda_{j}\right) \in[-\pi / 2, \pi / 2]$.

The state (2.2) is an eigenstate if the Bethe equations hold:

$$
\left(\frac{\sin \left(\lambda_{j}-i \eta / 2\right)}{\sin \left(\lambda_{j}+i \eta / 2\right)}\right)^{L} \prod_{k \neq j} \frac{\sin \left(\lambda_{j}-\lambda_{k}+i \eta\right)}{\sin \left(\lambda_{j}-\lambda_{k}-i \eta\right)}=1
$$

In this case the energy is given by

$$
E=\sum_{j} e\left(\lambda_{j}\right), \quad \text { where } \quad e(u)=\frac{4 \sinh ^{2} \eta}{\cos (2 u)-\cosh \eta}
$$

In this parametrization in the regime $\Delta>1$ the one-string solutions to the Bethe equations lie on the real axis. The Bethe equation also allows for the so-called $n$-string solutions that are centered on the real axis:

$$
\lambda_{k}=u+i \eta(n+1-2 k) / 2+\delta_{k} \quad k=1, \ldots, n
$$

where $u$ is the string center and $\delta_{k}$ are the string deviations that become exponentially small in the thermodynamic limit.

In the thermodynamic limit the solution to the Bethe equations can be characterized by root and hole densities $\rho_{n}(\lambda), \rho_{n}^{(h)}(\lambda)$ for the $n$-strings. It follows from the Bethe equations that these functions satisfy $\|3\|$

$$
\rho_{n}+\rho_{n}^{(h)}=\delta_{k, 1} s+s \star\left(\rho_{n-1}^{(h)}+\rho_{n+1}^{(h)}\right),
$$

\footnotetext{
${ }^{1}$ This follows from the fact that the finite volume overlap formulas arise from a set of algebraic manipulations on the Bethe Ansatz wave function, which has the same functional form for every $\Delta$. The differences between the regimes only show up at the solutions of the Bethe equations (including various types of singular rapidities [42]), and in the thermodynamic limit. However, these issues of the massless regime are not considered here.
} 
where

$$
s(u)=1+2 \sum_{n=1}^{\infty} \frac{\cos (2 n u)}{\cosh (\eta n)},
$$

and the convolution of two functions is defined as

$$
(f \star g)(u)=\int_{-\pi / 2}^{\pi / 2} \frac{d \omega}{2 \pi} f(u-\omega) g(\omega) .
$$

\subsection{Integrable initial states}

Given an initial state $\left|\Psi_{0}\right\rangle$ we are interested in the normalized squared overlaps

$$
\frac{\left|\left\langle\{\lambda\}_{N} \mid \Psi_{0}\right\rangle\right|^{2}}{\left\langle\{\lambda\}_{N} \mid\{\lambda\}_{N}\right\rangle}
$$

Before starting any calculations one has to decide which initial states to consider. The choice of $\left|\Psi_{0}\right\rangle$ can be motivated by their experimental relevance, their relation to other problems in mathematical physics [32, 34, 33], or their inherent integrability properties. In the present work we follow the latter approach and consider a special class of states that were called "integrable initial states" in the recent work [28. They include the Néel and dimer states, and other finitely entangled Matrix Product States (MPS's). These states are relevant both for an experimental realization and for the AdS/CFT conjecture. Nevertheless we concentrate on their integrability properties.

Integrable initial states are defined as states that are annihilated by all odd (with respect to space reflection) conserved charges of the model [28]:

$$
Q_{2 j+1}\left|\Psi_{0}\right\rangle=0 \text {. }
$$

Here the charges are defined in the usual way from the transfer matrix of the model [28], or alternatively, they can be generated in a formal way using the so-called boost operator 47. The eigenvalues of the charges on Bethe states are additive:

$$
Q_{2 j+1}\left|\left\{\lambda_{N}\right\}\right\rangle=\sum_{k=1}^{N} q_{2 j+1}\left(\lambda_{k}\right),
$$

where $q_{2 j+1}(\lambda)$ are known functions satisfying $q_{2 j+1}(\lambda)=-q_{2 j+1}(-\lambda)$. It follows from (2.11) that the only non-vanishing overlaps are those where the Bethe rapidities display the pair structure:

$$
\{\lambda\}_{N}=\left\{ \pm \lambda^{+}\right\} \cup\left\{\lambda^{\mathcal{S}}\right\}
$$

where $\lambda^{\mathcal{S}}$ are special rapidities for which $q_{2 j+1}\left(\lambda^{\mathcal{S}}\right)=0$. For $\Delta>1$ we have $\left\{\lambda^{\mathcal{S}}\right\} \subset\{0, \pi / 2\}$.

It was shown in 28 that a subclass of integrable states are those two-site states that are generated by local $K$-matrices of the boundary Algebraic Bethe Ansatz. The construction of [28] can be carried out for an arbitrary integrable spin chain with local dimension $d \geq 2$, if the $R$-matrix admits an appropriate crossing symmetry transformation. Integrable states are given as

$$
\left|\Psi_{0}\right\rangle=\otimes_{j=1}^{L / 2}|\psi\rangle
$$

where

$$
|\psi\rangle=\sum_{j_{1}, j_{2}=1}^{d}(K(-\eta / 2) V)_{j_{1}, j_{2}}\left|j_{1}\right\rangle \otimes\left|j_{2}\right\rangle,
$$

where $K(u)$ is a solution to the reflection equations 48, 49]

$$
R_{1,2}(u-w) K_{1}(u) R_{1,2}(u+w) K_{2}(w)=K_{2}(w) R_{1,2}(u+w) K_{1}(u) R_{1,2}(u-w),
$$

and the matrix $V$ is a similarity transformation describing crossing of the fundamental $R$ matrix:

$$
R_{1,2}^{t_{1}}(u)=\gamma(u) V_{1}^{-1} R_{1,2}(-u-\eta) V_{1}
$$


with $\gamma(u)$ being a known function, which depends on the overall normalization of the $R$ matrix. In the spin- $1 / 2$ chain we have $V=\sigma^{y}$ and the solutions to (2.16) form a 3-parameter family of matrices [50]. We use the parametrization

$$
\begin{aligned}
& K_{11}(u, \alpha, \beta, \theta)=2(\sinh (\alpha) \cosh (\beta) \cosh (u)+\cosh (\alpha) \sinh (\beta) \sinh (u)) \\
& K_{12}(u, \alpha, \beta, \theta)=e^{\theta} \sinh (2 u) \\
& K_{21}(u, \alpha, \beta, \theta)=e^{-\theta} \sinh (2 u) \\
& K_{22}(u, \alpha, \beta, \theta)=2(\sinh (\alpha) \cosh (\beta) \cosh (u)-\cosh (\alpha) \sinh (\beta) \sinh (u)) .
\end{aligned}
$$

It follows that every two-site product state is integrable, because for each $|\psi\rangle$ there are appropriate $(\alpha, \beta, \theta)$ parameters reproducing it (apart from the irrelevant overall phase and normalization) For the sake of completeness we give here the explicit formula for the twosite block:

$$
\begin{aligned}
& \psi_{11}(\alpha, \beta, \theta)=-e^{\theta} \sinh (\eta) \\
& \psi_{12}(\alpha, \beta, \theta)=2(-\sinh (\alpha) \cosh (\beta) \cosh (\eta / 2)+\cosh (\alpha) \sinh (\beta) \sinh (\eta / 2)) \\
& \psi_{21}(\alpha, \beta, \theta)=2(\sinh (\alpha) \cosh (\beta) \cosh (\eta / 2)+\cosh (\alpha) \sinh (\beta) \sinh (\eta / 2)) \\
& \psi_{22}(\alpha, \beta, \theta)=e^{-\theta} \sinh (\eta),
\end{aligned}
$$

where we neglected an irrelevant factor of $(i)$.

In 28] it was also shown that a wider class of integrable initial states can be generated by acting with the fundamental or fused transfer matrices on the two-site states. These states can be represented as Matrix Product States (MPS) with finite bond dimension, therefore they have finite entanglement and can approximate ground states of gapped Hamiltonians. However, these states will not be considered here, we will only focus on the local two-site states.

\subsection{Previous exact results for the finite volume overlaps}

Previous results in the literature concern the Néel and Dimer states, where the two-site blocks are given by

$$
\left|\psi_{\text {Néel }}\right\rangle=|\uparrow \downarrow\rangle \quad\left|\psi_{\text {Dimer }}\right\rangle=\frac{1}{\sqrt{2}}(|\uparrow \downarrow\rangle-|\downarrow \uparrow\rangle) .
$$

They belong to the class of generalized dimer states

$$
\left|\psi_{\gamma}\right\rangle \sim(|\uparrow \downarrow\rangle-\gamma|\downarrow \uparrow\rangle), \quad \gamma \in \mathbb{C} .
$$

These states are generated by the diagonal $K$-matrices, which can be obtained from (2.18) through the $\beta \rightarrow \infty$ limit. Alternatively, they can be described by the parametrization

$$
K(u, \xi)=\left(\begin{array}{cc}
\sinh (\xi+u) & 0 \\
0 & \sinh (\xi-u)
\end{array}\right) .
$$

It follows from $(2.15)$ that $\gamma=\frac{\sinh (\xi+\eta / 2)}{\sinh (\xi-\eta / 2)}$; the Néel and Dimer states are obtained by setting $\xi=-\eta / 2$ and $\xi=i \pi / 2$, respectively.

A special property of these states is that every two-site block has exactly one down spin, and so for non-vanishing overlaps the number of particles is always $N=L / 2$. Moreover, the structure of the overlaps is essentially the same for arbitrary $\xi$ : a simple argument 10,51 based on the coordinate Bethe Ansatz wave function shows that for generic $\gamma$

$$
\left\langle\Psi_{\gamma} \mid\{\lambda\}_{N}\right\rangle=\left\langle\Psi_{\text {Néel }} \mid\{\lambda\}_{N}\right\rangle \times \prod_{j=1}^{N} \frac{1+\gamma \frac{\sin \left(\lambda_{j}-i \eta / 2\right)}{\sin \left(\lambda_{j}+i \eta / 2\right)}}{\sqrt{1+|\gamma|}^{2}} .
$$

\footnotetext{
${ }^{2}$ It was shown in 28 that for other models (for example the spin-1 XXZ model) the local $K$-matrices produce only a subclass of two-site states, therefore generally not all two-site product states are integrable.
} 
Therefore, it is enough to determine the overlaps with the Néel state. The first results for this problem appeared in [10], where the following off-shell formula was derived for the unnormalized overlap:

$$
\left\langle\Psi_{\text {Néel }} \mid \lambda_{1}, \ldots, \lambda_{N}\right\rangle=\frac{\prod_{j} \sinh ^{L}\left(\lambda_{j}-\eta / 2\right) \sinh ^{L+1}\left(\lambda_{j}+\eta / 2\right)}{\prod_{j} \sinh \left(2 \lambda_{j}\right) \prod_{j<k} \sinh \left(\lambda_{j}-\lambda_{k}\right) \sinh \left(\lambda_{j}+\lambda_{k}\right)} \times \operatorname{det} L,
$$

where $L$ is a $N \times N$ matrix with elements given by

$$
L_{j k}=q_{2 j}\left(\lambda_{k}\right), \quad \text { where } \quad q_{a}(u)=\operatorname{coth}^{a}(u-\eta / 2)-\operatorname{coth}^{a}(u+\eta / 2) .
$$

It is important that (2.24) does not make use of the Bethe equations, and it merely represents an algebraic reorganization of the expression that can be obtained directly from the wave function (2.2). The idea behind the derivation of (2.24) is that the overlap can be related to a specific partition function of the six-vertex model, where the initial state $\left|\Psi_{0}\right\rangle$ plays the role of a reflecting boundary [10. For diagonal $K$-matrices this partition function was expressed by Tsushiya as a determinant [52, and (2.24) is a specific homogeneous limit of the Tsushiya determinant.

Despite its compact and explicit form, (2.24) is not convenient for practical purposes. First of all it is not clear how to perform the thermodynamic limit of the determinant, and second, the expression (2.24) becomes singular for the physically relevant cases (2.13).

A much more useful representation for on-shell states was obtained in 11, 12 based on an intermediate result for the Tsushiya determinant given in [35]. It was first shown in the work [11] that the overlap is non-vanishing only for states with the pair structure, and for these cases it was found that

$$
\frac{\left|\left\langle\Psi_{\text {Néel }} \mid\left\{ \pm \lambda^{+}\right\}_{N / 2}\right\rangle\right|^{2}}{\left\langle\left\{ \pm \lambda^{+}\right\}_{N / 2} \mid\left\{ \pm \lambda^{+}\right\}_{N / 2}\right\rangle}=\prod_{j=1}^{N / 2} v_{\text {Néel }}\left(\lambda_{j}\right) \times \frac{\operatorname{det}_{N / 2} G_{j k}^{+}}{\operatorname{det}_{N / 2} G_{j k}^{-}}
$$

where

$$
v_{\text {Néel }}(\lambda)=\frac{\tan \left(\lambda_{j}+i \eta / 2\right) \tan \left(\lambda_{j}-i \eta / 2\right)}{4 \sin ^{2}\left(2 \lambda_{j}\right)}
$$

and ${ }^{3}$

$$
G_{j k}^{ \pm}=\delta_{j k}\left(-L \varphi_{\eta / 2}\left(\lambda_{j}^{+}\right)+\sum_{l=1}^{L / 4} \varphi_{\eta}^{+}\left(\lambda_{j}^{+}, \lambda_{l}^{+}\right)\right)-\varphi_{\eta}^{ \pm}\left(\lambda_{j}^{+}, \lambda_{k}^{+}\right)
$$

with

$$
\begin{aligned}
\varphi_{\eta}^{ \pm}(\lambda, \mu) & =\varphi_{\eta}(\lambda-\mu) \pm \varphi_{\eta}(\lambda+\mu) \\
\varphi_{x}(\lambda) & =\frac{\sinh (2 x)}{\sinh (\lambda+i x) \sinh (\lambda-i x)} .
\end{aligned}
$$

In (2.26) it was assumed that $N$ is even and the Bethe state does not include the special rapidities $\left\{\lambda^{\mathcal{S}}\right\} \subset\{0, \pi / 2\}$; for those cases different regularized formulas are needed 12, 23.

Based on the identity (2.23) it is possible to derive the overlaps with the other states generated by diagonal $K$-matrices, and they all take the form of 2.26) with a different single particle overlap function. For example for the Dimer state we get [15, 51]

$$
v_{\text {Dimer }}(\lambda)=\frac{\sinh ^{4}(\eta / 2) \cot ^{2}(\lambda)}{\sin (2 \lambda+i \eta) \sin (2 \lambda-i \eta)} .
$$

\footnotetext{
${ }^{3}$ The matrix elements of $G^{ \pm}$include a sign difference as compared to the formulas given in 11, 12, but they agree with those of [53, 54. This sign does not influence the resulting overlaps. We introduced it in order to have positive elements in the diagonal of the matrix, and to avoid confusion with earlier results when taking the thermodynamic limit.
} 
Further overlap formulas were computed in the works 32, 34, 33. These papers considered certain Matrix Product States (MPS's) in the $S U(N)$ symmetric models, that are relevant for the AdS/CFT conjecture. Regarding the case of the spin-1/2 XXX chain real space calculations were used to derive the overlaps with Bethe states with a low number of particles, and formulas of the form $(2.26)$ were conjectured for higher particle numbers. It was shown in 28 that the MPS's in question are in fact integrable, and they can be identified either as zeromomentum components of specific two-site states, or they result as action of transfer matrices on such states. Exact overlap formulas were derived in [34], which considered overlaps with the so-called partial Néel states. They are zero-momentum components of two-site states where $\psi_{11}=0$. According to (2.19) they correspond to a special scaling limit, where the $K$ matrix becomes lower diagonal. For these cases an exact off-shell formula was derived, that is closely related to (2.24), with a modified matrix. However, in the on-shell case an exact result of the form (2.26) was obtained once again. The work 34 used a slightly modified version of the argument of Tsushiya [52 to obtain the off-shell result, but it was demonstrated in [55] that it is not evident how to apply this idea to the generic case with $\psi_{11} \neq 0$.

The results of [32, 34, 33] seem to indicate that the on-shell overlaps are always of the form (2.26), even for off-diagonal $K$-matrices. However, this is somewhat misleading. These works only consider the $S U(2)$ invariant chain, where every $K$ matrix can be rotated to diagonal one. It is known that the Bethe vectors are highest weight states and that the spin lowering operators can be represented with infinite rapidity particles. Therefore, the $S U(2)$ rotations can be evaluated on the Bethe vectors, and overlaps with off-diagonal $K$-matrices can be expressed with those of the known diagonal cases. This procedure is analogous to that used in 13 to study $q$-raised Néel states in the XXZ model. Nevertheless we believe that the method of [34] could be applied to lower diagonal $K$-matrices even in the XXZ case, which would then yield genuine new rigorous results.

Further results appeared in [27], which considered MPS's for higher rank models. In the $S U(3)$ case formulas analogous to 2.26 were obtained from coordinate Bethe Ansatz, with the determinants mirroring the nested Bethe Ansatz solution of the model. The resulting overlap formulas were used in 26 to study the corresponding quench situation, and it was later shown in 28 that the initial state is in fact integrable. On the other hand, the interpretation of these results within Boundary Algebraic Bethe Ansatz is not yet known, and this is an intriguing problem to be investigated in future work. However, in the present paper we content ourselves with the open questions of the spin- $1 / 2 \mathrm{XXZ}$ chain.

\subsection{The Quench Action method}

The Quench Action (QA) method [9] was devised to determine which Bethe states are relevant for the long-time behavior of physical observables in quantum quenches. The essence is to investigate the overlap sum rule

$$
1=\left\langle\Psi_{0} \mid \Psi_{0}\right\rangle=\sum_{\{\lambda\}} \frac{\left|\left\langle\Psi_{0} \mid\{\lambda\}\right\rangle\right|^{2}}{\langle\{\lambda\} \mid\{\lambda\}\rangle},
$$

where the summation runs over all eigenstates of a finite volume system, and to select the states that dominate the sum in the thermodynamic limit. It can be argued that the same states determine the long-time averages of local observables [9]. The selection of the relevant states is achieved by transforming the finite sum into a functional integral over root densities, and minimizing the resulting Quench Action, which is defined as the combination of the overlap and entropy of a state with a given root distribution. The QA is completely analogous to the free energy functional of the finite temperature situation, therefore the resulting equations always take the form of (generalized) Thermodynamic Bethe Ansatz (TBA) equations [3].

Before exchanging the summation for a functional integral it is important to remember that for the integrable quenches considered here the non-vanishing terms in the sum (2.31) are the parity invariant Bethe states. Therefore, these states can be completely determined by listing the positive rapidities (defined either as $\Re\left(\lambda^{+}\right)>0$ for all roots, or $\lambda_{n}^{+}>0$ for 
the string centers), and this affects the entropy associated to a given root configuration. For the $\mathcal{O}(L)$ term of the entropy in the QA we get a factor of $1 / 2$ as compared to the formulas of the usual TBA. In the next subsection we also investigate the $\mathcal{O}(1)$ pieces, but first we collect the known formulas for the leading part.

In [9] it was shown that the sum (2.31) leads to the functional integral

$$
\int D \rho_{n}\left(\lambda^{+}\right) e^{-S_{Q A}\left(\left\{\rho_{n}(\lambda)\right\}\right)}
$$

with

$$
S_{Q A}\left(\left\{\rho_{n}(\lambda)\right\}\right)=-2 \Re \ln \left\langle\Psi_{0} \mid\left\{\rho_{n}(\lambda)\right\}\right\rangle+\frac{1}{2} S_{Y Y}\left(\left\{\rho_{n}(\lambda)\right\}\right)
$$

with $S_{Y Y}$ being the Yang-Yang entropy

$$
S_{Y Y}\left(\rho_{n}(\lambda)\right) \equiv L \sum_{n=1}^{\infty} \int_{-\infty}^{+\infty} d \lambda\left[\rho_{n}(\lambda) \ln \left(1+\frac{\rho_{n}^{h}(\lambda)}{\rho_{n}(\lambda)}\right)+\rho_{n}^{h}(\lambda) \ln \left(1+\frac{\rho_{n}(\lambda)}{\rho_{n}^{h}(\lambda)}\right)\right] .
$$

The factor of $1 / 2$ in front of $S_{Y Y}$ results from the aforementioned restriction on parity invariant states.

In writing down 2.33 we assumed that the overlap is a known smooth function of the root densities. In particular, the TBA formalism can be applied if it can be written as

$$
\lim _{L \rightarrow \infty} \frac{1}{L} 2 \Re \ln \left\langle\Psi_{0} \mid\left\{\rho_{n}(\lambda)\right\}\right\rangle=\sum_{n=1}^{\infty} \int d \lambda \rho_{n}(\lambda) g_{n}(\lambda),
$$

with $g_{n}(\lambda)$ being the $n$-string overlap functions. If 2.35 ) holds for most Bethe states in the thermodynamic limit (TDL), then the minimization of the Quench Action leads to the following set of generalized TBA equations 14,19 :

$$
\log Y_{j}=d_{j}+s \star\left[\log \left(1+Y_{j-1}\right)+\log \left(1+Y_{j+1}\right)\right]
$$

where $Y_{j}(\lambda)=\rho_{j}^{(h)}(\lambda) / \rho_{j}(\lambda)$ and

$$
d_{j}=-g_{j}+s \star\left(g_{j-1}+g_{j+1}\right), \text { with } g_{0}=0 .
$$

Supplied with asymptotic conditions on the growth of $Y_{j}$ as $j \rightarrow \infty$ these equations completely determine the root densities.

We note that for a generic initial state there would be no guarantee that the overlaps lead to the form (2.35) in the TDL, and even if they do, the $g_{n}(\lambda)$ functions could be algebraically independent from each other. In fact, it is known that an analogous situation happens for non-integrable states in the framework of GGE $[19]$. On the other hand, a relatively simple situation arises if the overlap factorizes in finite volume.

We call an overlap factorizable if for on-shell states it can be written as

$$
\frac{\left|\left\langle\Psi_{0} \mid\left\{ \pm \lambda^{+}\right\}_{N / 2}\right\rangle\right|^{2}}{\left\langle\left\{ \pm \lambda^{+}\right\}_{N / 2} \mid\left\{ \pm \lambda^{+}\right\}_{N / 2}\right\rangle}=A^{L} \prod_{j=1}^{N / 2} v\left(\lambda_{j}^{+}\right) \times C(L, N,\{\lambda\}),
$$

where $C(L, N,\{\lambda\})$ is a function that remains $\mathcal{O}\left(L^{0}\right)$ in the thermodynamic limit. The known exact results presented in subsection 2.3 were all factorizable. The separate pre-factor $A^{L}$ was not present in previous results, but it can be argued that such a factor necessarily appears for initial states having components with non-zero magnetization, in which cases the particle number is independent of $L$. In (2.38) we assumed the pair structure; we will argue below that factorizability is a property that indeed only holds for integrable initial states.

It is important that the formula (2.38) represents factorization on the level of the individual rapidities. For the string contributions in 2.35 ) we obtain

$$
g_{n}(\lambda)=-\sum_{k=1}^{n} \log (v(\lambda+i \eta(n+1-2 k) / 2)),
$$


and this specifies the sources in (2.36) through (2.37). As a consequence, it can be shown that the solutions of the QA-TBA always satisfy the $Y$-system equations

$$
Y_{j}(\lambda+i \eta / 2) Y_{j}(\lambda-i \eta / 2)=\left(1+Y_{j-1}(\lambda)\right)\left(1+Y_{j+1}(\lambda)\right),
$$

The proof is straightforward by combining (2.36), 2.37) and (2.39) and making use of the relation

$$
\lim _{\delta \rightarrow \eta / 2}\left[\int_{-\pi / 2}^{\pi / 2} \frac{d x}{2 \pi}(s(y+i \delta-x)+s(y-i \delta-x)) f(x)\right]=f(y),
$$

which follows from the integral representation (2.8) and is valid for any smooth function $f(x)$.

The Y-system is regarded as an important sign of integrability of the initial state, and it was used as a tool for finding exact solution to the TBA [14, 21]. In the Quench Action framework the Y-system ultimately follows from factorizability of the overlaps, which is expected to be a general property of integrable initial states 28 . Conversely, in the generic case where the $Y$-system does not hold [17, 19], the overlaps with the initial state can not have the factorized form.

\subsection{The overlap sum rule}

It is worthwhile to investigate the overlap sum rule (2.31) in more detail, which sheds some light on the role of the Gaudin-like determinants in the overlaps. To this order we give a slightly different definition of the Quench Action.

In analogy with the partition functions in thermodynamics we define

$$
S_{Q A}=-\log \sum_{\left\{ \pm \lambda^{+}\right\}} \frac{\left|\left\langle\Psi_{0} \mid\left\{ \pm \lambda^{+}\right\}_{N / 2}\right\rangle\right|^{2}}{\left\langle\left\{ \pm \lambda^{+}\right\}_{N / 2} \mid\left\{ \pm \lambda^{+}\right\}_{N / 2}\right\rangle}
$$

with a large volume behavior given by

$$
S_{Q A}=s_{Q A} L+\Delta S+\mathcal{O}\left(L^{-1}\right)
$$

where $s_{Q A}$ is the QA density, and $\Delta S$ is an $\mathcal{O}(1)$ piece that has not yet been investigated in the literature. From the definition we have the trivial identity $S_{Q A}=0$, and our goal is to derive this within the Quench Action method, both for the leading and the sub-leading part.

The extensive part is given by (2.33)-2.35) evaluated at the saddle point solution. It was derived in [15, 51] that

$$
s_{Q A}=\int_{-\pi / 2}^{\pi / 2} \frac{d \lambda}{2 \pi} s(\lambda)\left[\log \left(1+Y_{1}(\lambda)\right)+\log (v(\lambda))\right],
$$

where $Y_{j}(\lambda)$ is the solution of the QA-TBA (2.36) and $v(\lambda)$ is the single-particle overlap function. Here it was assumed that the initial state has zero total magnetization $\|^{\Uparrow}$ the formula is analogous to the expression of the free energy density within the usual TBA [3]. In $[15,51$ the relation $s_{Q A}=0$ was used as a consistency condition within the QA; it also served as a test for the accuracy of the numerical solution of the TBA equations.

In order to determine the finite term $\Delta S$ we first investigate the thermodynamic limit of the overlap formula $(2.26)$. Previously we have treated the extensive part, which leads to 2.35 ) with the $n$-string overlaps given by (2.39). On the other hand, the ratio of the determinants in (2.26) gives a non-zero $\mathcal{O}(1)$ contribution. In order to evaluate this term the first step is to express the ratio of determinants using only the string centers. This task is analogous to finding the norm of Bethe states with strings $[56]$, and for the determinants in question it was performed in 23]. Denoting by $\lambda_{n, a}$ the $n$-string centers with index $a$ we get

$$
\frac{\operatorname{det} G^{+}}{\operatorname{det} G^{-}} \rightarrow \frac{\operatorname{det} \tilde{G}^{+}}{\operatorname{det} \tilde{G}^{-}}
$$

\footnotetext{
${ }^{4}$ The generic case was not discussed in [15, 51], because existing overlap formulas were only available for initial states with zero total magnetization.
} 
with

$$
\tilde{G}_{(n, a),(m, b)}^{ \pm}=\delta_{(n, a),(m, b)}\left(-L \varphi_{n \eta / 2}\left(\lambda_{j}\right)+\sum_{(o, c)} \Theta_{n, o}^{+}\left(\lambda_{n, a}, \lambda_{o, c}\right)\right)-\Theta_{n, m}^{ \pm}\left(\lambda_{n, a}, \lambda_{m, b}\right),
$$

where

$$
\begin{gathered}
\Theta_{n, m}^{ \pm}(\lambda, \mu)=\Theta_{n, m}(\lambda-\mu) \pm \Theta_{n, m}(\lambda+\mu) \\
\Theta_{n, m}(\lambda)= \begin{cases}\varphi_{|n-m| \eta / 2}(\lambda)+\sum_{j=1}^{(n+m-|n-m|-1) / 2} \varphi_{(|n-m|+2 j) \eta / 2}(\lambda)+\varphi_{|n+m| \eta / 2}(\lambda) & \text { if } n \neq m \\
\sum_{j=1}^{n-1} 2 \varphi_{j \eta}(\lambda)+\varphi_{n \eta}(\lambda) & \text { if } n=m\end{cases}
\end{gathered}
$$

and $\varphi_{x}(\lambda)$ is given in 2.29$)$.

In the thermodynamic limit the ratio of the two determinants leads to a ratio of two Fredholm determinants. The calculation proceeds through standard steps [57, 54, 58] and here we only give the main result. In the TDL we get

$$
\lim _{L \rightarrow \infty} \frac{\operatorname{det} \tilde{G}^{+}}{\operatorname{det} \tilde{G}^{-}}=\frac{\operatorname{det}\left(1-\hat{Q}^{+}\right)}{\operatorname{det}\left(1-\hat{Q}^{-}\right)}
$$

where $\hat{Q}^{ \pm}$are integral operators that act on functions $f_{n}(\lambda)$ with a string index $n$ defined for $\lambda \in \mathbb{R}^{+}$. The action of the integral operators reads

$$
\left(\hat{Q}^{ \pm}(f)\right)_{n}(x)=\sum_{m=1}^{\infty} \int_{0}^{\infty} \frac{d y}{2 \pi}\left(\Theta_{n, m}^{ \pm}(x, y)\right) \frac{1}{1+Y_{m}(y)} f_{m}(y) .
$$

The second step to calculate the $\mathcal{O}(1)$ terms in the sum rule is to carefully consider the summation over the eigenstates and the transformation of the sum into a functional integral. It was explained in the papers [39, 40| (inspired by the earlier work |59|) that in such situations there are two $\mathcal{O}(1)$ contributions: one coming from the density of states in rapidity space (corresponding to the change of variables from the integer momentum quantum numbers to the rapidities) and one from the quadratic fluctuations around the saddle point solution. The two terms depend on the nature of the Bethe equations. In the usual TBA setting with periodic boundary conditions the two contributions just cancel each other, whereas in a system with boundaries they combine to a finite and well defined term. In the overlap sum rule the only allowed states are those with the pair structure, and this corresponds formally to the boundary case, because both the density of states and the variations around the saddle point have to be calculated by varying only half of the rapidities. Therefore, the boundary results of [39, 40] apply, which read

$$
\sum_{\left\{\lambda^{+},-\lambda^{+}\right\}} \rightarrow \frac{\operatorname{det}\left(1-\hat{Q}^{-}\right)}{\operatorname{det}\left(1-\hat{Q}^{+}\right)} \int D \rho_{n}\left(\lambda^{+}\right),
$$

where $\hat{Q}^{ \pm}$are the same Fredholm that appeared as the limits of the Gaudin-like determinants. We note that the papers [39, 40] did not discuss theories with multiple particle species (such as the strings in the XXZ chain), however the generalization of the results given there is straightforward.

Combining the previous results we find that

$$
e^{-\Delta S}=\frac{\operatorname{det}\left(1-\hat{Q}^{-}\right)}{\operatorname{det}\left(1-\hat{Q}^{+}\right)} \times \frac{\operatorname{det}\left(1-\hat{Q}^{+}\right)}{\operatorname{det}\left(1-\hat{Q}^{-}\right)}=1,
$$

as required by definition. Thus the ratio of the two Gaudin-like determinants ensures that the overlaps have the correct normalization in the thermodynamic limit. We believe that this has not been noticed in earlier works. 
The present result applies to the states 2.21 generated by the diagonal $K$-matrices, where the overlap of the form $(2.26)$ is rigorously proven. It is remarkable, that the $\mathcal{O}(1)$ terms do not depend on the free parameter of the initial state: for the overlap this follows from relation (2.23), whereas for the normalization of the functional integral it is a result of the pair structure. This leads to the conjecture, that the same Gaudin-like determinants should appear also for the generic $K$-matrices; this conjecture and its tests are presented in Section 3. In the Conclusions we discuss further implications of these observations.

\subsection{Calculation of the Loschmidt echo}

An important property of integrable two-site states is that the extensive part of the Loschmidt amplitude (also called the dynamical free energy) can be computed analytically. The Loschmidt amplitude is defined as the overlap of a time evolved state with the original initial state:

$$
L(t)=\left\langle\Psi_{0}\left|e^{-i H t}\right| \Psi_{0}\right\rangle,
$$

and the dynamical free energy is defined as

$$
g(w)=-\lim _{L \rightarrow \infty} \frac{1}{L} \log L(-i w) .
$$

It was shown in 60, 41 that these quantities can be evaluated by a lattice path integral, where the initial and final states $\left\langle\Psi_{0}\right|$ and $\left|\Psi_{0}\right\rangle$ play the role of boundary conditions. The corresponding partition function can be evaluated in the rotated channel by the so-called Boundary Quantum Transfer Matrix (QTM), if the two site block $|\psi\rangle$ is identified with two different $K$-matrices $K_{ \pm}\left(u, \alpha_{ \pm}, \beta_{ \pm}, \theta_{ \pm}\right)$as

$$
|\psi\rangle \sim \sum_{j_{1}, j_{2}=1}^{2}\left(K_{-}(-\eta / 2) \sigma^{y}\right)_{j_{1}, j_{2}}\left|j_{1}\right\rangle \otimes\left|j_{2}\right\rangle, \quad(|\psi\rangle)^{*} \sim \sum_{j_{1}, j_{2}=1}^{2}\left(\tilde{K}_{+}(\eta / 2) \sigma^{y}\right)_{j_{1}, j_{2}}\left|j_{1}\right\rangle \otimes\left|j_{2}\right\rangle,
$$

where $\tilde{K}_{+}$denotes transposition. It can be seen from (2.18) that the two vectors in $(2.55)$ can be proportional to each other only if the two sets of parameters satisfy

$$
\alpha_{-}=-\alpha_{+}^{*} \equiv \alpha \quad \beta_{-}=\beta_{+}^{*} \equiv \beta \quad \theta_{-}=-\theta_{+}^{*} \equiv \theta .
$$

In the thermodynamic limit the amplitude (2.53) will be given by the leading eigenavalue of the Boundary QTM, for which the following generalized TBA equations were derived in |41|⿴囗十⺝

$$
\log \tilde{y}_{j}=-4 \sinh (\eta) w s \delta_{j, 1}+\tilde{d}_{j}+s \star\left[\log \left(1+\tilde{y}_{j-1}\right)+\log \left(1+\tilde{y}_{j+1}\right)\right],
$$

such that the dynamical free energy is given by

$$
g(w)=\frac{1}{2} \int_{-\pi / 2}^{+\pi / 2} d \lambda s(\lambda)\left\{w 2 \sinh (\eta) a(\lambda)+\log \left[\frac{1+\tilde{y}_{1}(\mu)}{1+\tilde{Y}_{1}(\lambda)}\right]\right\},
$$

where

$$
a(\lambda)=\frac{4 \sinh (\eta)}{\cosh (\eta)-\cos (2 \lambda)} .
$$

and $\tilde{Y}_{j}(\lambda)$ is the solution of $(2.57)$ for $w=0$. The source terms $\tilde{d}_{j}$ are independent of $w$ and are determined by the parameters of the $K$-matrices. A number of specific cases were discussed in [41, but we refrain from repeating the explicit formulas. Equation (2.57) will be called "Loschmit-TBA" in the rest of the paper.

\footnotetext{
${ }^{5}$ The formulas here have an additional factor of 4 in front of the energy terms; which originates from a different definition of the Hamiltonian.
} 
An important central result of [41] was that the functions $\tilde{Y}_{j}$ satisfy the $Y$-system equations, which follows from the fusion hierarchy of the Boundary QTM. Also, it was shown that the first member is given explicitly by

$$
1+Y_{1}(\lambda)=\frac{\mathcal{N}(\lambda+i \eta / 2) \mathcal{N}(\lambda-i \eta / 2)}{\chi(\lambda)}
$$

where

$$
\mathcal{N}(\lambda)=\operatorname{Tr}\left[K_{+}(\lambda+\eta / 2) K_{-}(\lambda-\eta / 2)\right]
$$

and

$$
\chi=16 \frac{v_{\eta}^{s} v_{\eta}^{c}}{v_{\eta / 2}^{s} v_{\eta / 2}^{c}} v_{\alpha}^{s} v_{\alpha^{*}}^{s} v_{\beta}^{c} v_{\beta^{*}}^{c}
$$

where we introduced the short-hand notation

$$
v_{\kappa}^{s}(\lambda)=\sin (\lambda+i \kappa) \sin (\lambda-i \kappa) \quad v_{\kappa}^{c}(\lambda)=\cos (\lambda+i \kappa) \cos (\lambda-i \kappa)
$$

Note that according to $2.55 \mathcal{N}(0)$ describes the norm of the two-site block $|\psi\rangle$.

Based on the similarities of the QA formulas (2.36) and those obtained for the Loschmidt echo it is very natural to identify

$$
\tilde{Y}_{j}(\lambda)=Y_{j}(\lambda) \quad \tilde{d}_{j}(\lambda)=d_{j}(\lambda),
$$

which was known to hold for the diagonal $K$ matrices [51] and was also checked explicitly for specific off-diagonal cases in 41. Eq. (2.64) represents a close connection between the QA and QTM approaches: it implies that the integrability properties of the overlaps (i.e. factorizability) are closely related to the fusion hierarchy of the Boundary QTM. This is the observation that allows us to extract new finite volume overlap formulas from the LoschmidtTBA.

\section{The general overlap formula}

In the previous section it was explained that (2.57) is a generalized TBA equation describing the Loschmidt-echo, valid for arbitrary $K$-matrices, such that the solution at $w=0$ (corresponding to the Quench Action point) is exactly known through (2.60). If we assume that there exists a factorized overlap of the form (2.38), then (2.57) has to coincide with the QA-TBA 2.36) for that specific overlap. This is supported by the identification (2.64). In this case the single-particle overlap function can be ,reverse engineered” by computing the overlap sources $d_{j}(u)$ in (2.36) and comparing them to $\tilde{d}_{j}(u)$ in (2.57). Instead of computing the source terms, we choose to operate only with the $Y$-functions and their singularity properties.

For example, for the first equation in the TBA we obtain the suggestive formula

$$
\begin{aligned}
\log Y_{1}(\lambda)-s \star & {\left[\log \left(Y_{1}(\lambda+i \eta / 2)\right)+\log \left(Y_{1}(\lambda-i \eta / 2)\right)\right]=} \\
& =-\log (v(\lambda))+s \star[\log (v(\lambda+i \eta / 2))+\log (v(\lambda-i \eta / 2))] .
\end{aligned}
$$

Here we used the $Y$-system equations (2.40), and relations (2.37) and (2.39) for the overlaps. It is tempting to identify $v(\lambda)=C / Y_{1}(\lambda)$, however, this is misleading. Relation (3.1) gives information only about the poles and zeroes of the functions within the physical strip $|\Im(\lambda)| \leq$ $\eta / 2$. This follows from the identity

$$
\int_{-\pi / 2}^{\pi / 2} \frac{d x}{2 \pi} s(y-x) \log (h(x+i \eta / 2) h(x-i \eta / 2))=\log h(y),
$$

\footnotetext{
${ }^{6}$ The most general case with arbitrary complex $(\alpha, \beta, \theta)$ parameters was not given in 41. However, it is straightforward to extract it from the intermediate results given there.
} 
which can be obtained from the Fourier representation of $s(\lambda)(2.8)$, or alternatively after a contour shift from (2.41), assuming that there are no singularities of $\log (h(x))$ to be picked up. Therefore, (3.1) implies that $v(\lambda)=h(\lambda) / Y_{1}(\lambda)$, where $h(\lambda)$ is free of poles and zeroes in the physical strip, and the poles (or zeroes) of $Y_{1}(\lambda)$ within the physical strip correspond to zeroes (or poles) of $v(\lambda)$, respectively.

In order to understand the dependence of the overlaps on the $K$-matrices it is useful to study the known cases. For a diagonal $K$-matrix of the form $(2.22)$ the overlap function is

$$
v(\lambda)=\frac{\sinh ^{4}(\eta)}{16(\cosh (2 \xi) \cosh (\eta)-1)^{2}} \frac{\left(v_{\xi}^{s}\right)^{2}}{v_{0}^{s} v_{0}^{c} v_{\eta / 2}^{s} v_{\eta / 2}^{c}},
$$

which is obtained from the relation (2.23) and the Néel overlap function (2.27). For $Y_{1}(u)$ the following result holds:

$$
1+Y_{1}(\lambda)=\left(1+\mathfrak{a}(\lambda+i \eta / 2)\left(1+\mathfrak{a}^{-1}(\lambda-i \eta / 2)\right)\right.
$$

with

$$
\mathfrak{a}(\lambda)=\frac{\sin (\lambda-i(\xi-\eta / 2))}{\sin (\lambda+i(\xi-\eta / 2))} \frac{\sin (\lambda+i(\xi+\eta / 2))}{\sin (\lambda-i(\xi+\eta / 2))} \frac{\sin (2 \lambda-i \eta)}{\sin (2 \lambda+i \eta)} .
$$

Eq. (3.4) could be obtained by taking the diagonal limit of the general formula (2.60), or from earlier results for the diagonal $K$-matrices [60, 51]. It can be seen that the only poles of $Y_{1}(\lambda)$ within the physical strip can be at $\lambda= \pm \xi$, if $\Re(\xi)<\eta / 2$, and these poles are indeed reflected by the zeroes of $v(\lambda)$. In the cases where $\xi$ is outside the physical strip, the two singularities still move together, which is ensured by analytic continuation. The poles of $v(\lambda)$ within the physical strip are the double poles at $\lambda=0, \pi / 2$, and direct calculation shows that indeed they correspond to zeroes of $Y_{1}(\lambda)$. There are additional poles of both $v(u)$ and $Y_{1}(\lambda)$ at $\lambda= \pm i \eta / 2$ and $\lambda=\pi / 2 \pm i \eta / 2$, but they lie on the boundary of the physical strip, and (3.1) does not give any information about them: the contributions of symmetrically located singularities at $\kappa \pm i \eta / 2$ cancel each other in (3.1) for any $\kappa \in \mathbb{R}$, but they are relevant for the higher nodes of the TBA. The crucial observation here is that there are some fixed ( $\xi$-independent) singularities of both functions, and extra zeroes of $v(\lambda)$ that are determined by the $\xi$-dependent poles of $Y_{1}(\lambda)$. This is enough to conjecture the overlap function for the general case.

For a generic $K$-matrix the poles of $Y_{1}$ are given by (2.62), and comparing to the previous result (3.3) we arrive at the conjecture

$$
v(\lambda)=C u(\lambda), \quad u(\lambda)=\frac{v_{\alpha}^{s} v_{\alpha^{*}}^{s} v_{\beta}^{c} v_{\beta^{*}}^{c}}{v_{\eta / 2}^{s} v_{\eta / 2}^{c} v_{0}^{s} v_{0}^{c}}
$$

where $C$ is a numerical constant that depends on the parameters $(\alpha, \beta, \theta)$. A detailed analysis of all higher nodes in the TBA reveals that this overlap function indeed reproduces the correct source terms. We note that (3.6) does not include any "minimal analyticity assumption": any other function $\tilde{v}(\lambda)$ has to have exactly the same poles and zeroes as $v(\lambda)$ in order to yield the correct Loschmidt-TBA, and combined with the symmetry properties we get $\tilde{v}(\lambda)=\tilde{C} v(\lambda)$.

The overall normalization plays a similar role as a magnetic field in the thermodynamics: it influences the net magnetization. For states with zero total magnetization the constant $C$ can be fixed by the overlap sum rule. In these cases we expect that $A=1$ in (2.38), and the extensive part of the overlap sum rule (2.44) gives

$$
\int_{-\pi / 2}^{\pi / 2} \frac{d \lambda}{2 \pi} s(\lambda) \log \left[\mathcal{N}(\lambda+i \eta / 2) \mathcal{N}(\lambda-i \eta / 2) \frac{C(\alpha, \xi)}{16 v_{\eta}^{s}(\lambda) v_{\eta}^{c}(\lambda) v_{0}^{s}(\lambda) v_{0}^{c}(\lambda)}\right]=0 .
$$

Substituting $h(\lambda)=\sin (2 \lambda+i \eta) \sin (2 \lambda-i \eta)$ into 3.2 we get

$$
\int_{-\pi / 2}^{\pi / 2} \frac{d \lambda}{2 \pi} s(\lambda) \log \frac{1}{16 v_{\eta}^{s}(\lambda) v_{\eta}^{c}(\lambda) v_{0}^{s}(\lambda) v_{0}^{c}(\lambda)}=-\log \sinh ^{2}(\eta) .
$$


It can be checked that there are no zeros of $\mathcal{N}$ within the physical strip (this is most easily checked for the diagonal case (2.22) ), which implies

$$
\int_{-\pi / 2}^{\pi / 2} \frac{d \lambda}{2 \pi} s(\lambda) \log (\mathcal{N}(\lambda+i \eta / 2) \mathcal{N}(\lambda-i \eta / 2))=\log \mathcal{N}(0) .
$$

From this we get the result

$$
C(\alpha, \beta, \theta)=\frac{\sinh ^{4}(\eta)}{\mathcal{N}^{2}(0)} .
$$

It is important that the previous arguments only fix the thermodynamic part of the overlap. On the other hand, Section 2.5 showed that the two Gaudin-like determinants produce just the correct normalization in order to satisfy the overlap sum rule, and the $\mathcal{O}(1)$ terms do not depend on the extensive part, they are fixed simply by the pair structure. Therefore it is tempting to assume that the finite part is always given by the ratio of the same Gaudin-like determinants. This leads to the general finite volume conjecture

$$
\frac{\left|\left\langle\Psi_{0} \mid\left\{ \pm \lambda^{+}\right\}_{N / 2}\right\rangle\right|^{2}}{\left\langle\left\{ \pm \lambda^{+}\right\}_{N / 2} \mid\left\{ \pm \lambda^{+}\right\}_{N / 2}\right\rangle}=\tilde{A}^{L-2 N} \prod_{j=1}^{N / 2} v\left(\lambda_{j}^{+}\right) \times \frac{\operatorname{det}_{N / 2} G_{j k}^{+}}{\operatorname{det}_{N / 2} G_{j k}^{-}},
$$

with the matrices given by (2.28). As remarked in Sec. 2.4, the pre-factor $\tilde{A}^{L-2 N}$ can be present for states with non-zero total magnetization. If formula (3.11) is correct, then it should also apply to the zero particle case, which fixes $\tilde{A}$ :

$$
\tilde{A}=\frac{\left|\psi_{11}\right|}{|\psi|}=\frac{\left|K_{12}^{-}(-\eta / 2)\right|}{\sqrt{|\mathcal{N}(0)|}} .
$$

Combined with (2.19) this gives the remarkably simple formula

$$
\frac{\left|\left\langle\Psi_{0} \mid\left\{ \pm \lambda^{+}\right\}_{N / 2}\right\rangle\right|^{2}}{\left\langle\left\{ \pm \lambda^{+}\right\}_{N / 2} \mid\left\{ \pm \lambda^{+}\right\}_{N / 2}\right\rangle}=\frac{\left|e^{\theta(L-2 N)}\right| \sinh ^{L}(\eta)}{|\mathcal{N}(0)|^{L / 2}} \prod_{j=1}^{N / 2} u\left(\lambda_{j}\right) \times \frac{\operatorname{det}_{N / 2} G_{j k}^{+}}{\operatorname{det}_{N / 2} G_{j k}^{-}},
$$

with $u(\lambda)$ given by (3.6).

It is useful to give a few comments about the formula (3.13). The denominator $|\mathcal{N}(0)|^{L / 2}$ can be interpreted as an overall normalization factor coming from the norm of a single twosite block $|\psi\rangle$, as given by (2.15). The $\theta$ parameter of the $K$-matrix does not appear in the function $u(\lambda)$, but it affects the norm $\mathcal{N}(0)$ and it appears in a separate pre-factor. This factor can be easily understood from the coordinate Bethe Ansatz: it can be seen from (2.19) that in the un-normalized vector $\left|\Psi_{0}\right\rangle$ the up/down spin components carry factors of $e^{ \pm \theta / 2}$, respectively; the Bethe vectors have fixed magnetization, therefore these factors multiply to the common pre-factor in (3.13). It is also useful to investigate the diagonal limit of (3.13), which is reached by sending $\beta \rightarrow \infty$. In this limit both $\mathcal{N}(0)$ and $u(\lambda)$ diverge. It can be seen that the overlap scales to zero unless $N=L / 2$, in which case the previous results are reproduced.

We have tested the conjectured formula numerically and found convincing agreement in all cases. A short discussion of the numerical results is presented in the Appendix. In the remainder of the section we compute a few specific cases for the overlap.

The first example is the tilted ferromagnetic state defined as

$$
|\Psi(F, \theta)\rangle=\prod_{j=1}^{L} \frac{1}{\sqrt{2 \cosh (\theta)}}\left(\begin{array}{c}
i e^{\theta / 2} \\
e^{-\theta / 2}
\end{array}\right) .
$$

Here $\theta=\log (\cot (\vartheta / 2))$ with $\vartheta$ being the tilting angle from the $z$-axis. This state is obtained from (2.19) by setting

$$
\alpha=0 \quad \beta=\eta / 2+i \pi / 2 .
$$


In this case

$$
\mathcal{N}(0)=-4 \sinh ^{2}(\eta) \cosh ^{2}(\theta)
$$

Thus we get

$$
\frac{\left|\left\langle\Psi(F, \theta) \mid\left\{ \pm \lambda^{+}\right\}_{N / 2}\right\rangle\right|^{2}}{\left\langle\left\{ \pm \lambda^{+}\right\}_{N / 2} \mid\left\{ \pm \lambda^{+}\right\}_{N / 2}\right\rangle}=\frac{\mid e^{\theta(L-2 N) \mid}}{|2 \cosh (\theta)|^{L}} \prod_{j=1}^{N / 2} u_{F}\left(\lambda_{j}\right) \times \frac{\operatorname{det}_{N / 2} G_{j k}^{+}}{\operatorname{det}_{N / 2} G_{j k}^{-}}
$$

with

$$
u_{F}(\lambda)=\tan ^{2}(\lambda) \tan (\lambda+i \eta / 2) \tan (\lambda-i \eta / 2) .
$$

It is an interesting idea to consider the tilted ferromagnetic states in spin chains with an odd number of sites. In these cases the integrability properties (or possible off-shell formulas) can not follow from Boundary Bethe Ansatz methods, because those imply an even number of sites. However, these one-site states are integrable even in odd volumes: they are annihilated by all odd charges, which follows simply from the additivity of the charges and the fact that the states are integrable in even volumes. Therefore, the overlaps satisfy the pair structure also in odd volumes. We have tested the formula (3.17) in odd volumes and found that it is indeed correct. This points to the possibility of a derivation that is independent of the Boundary BA techniques.

Our second example is the tilted Néel state $|\Psi(N, \vartheta)\rangle$ given by the two-site block

$$
|\psi\rangle \sim\left(\begin{array}{cc}
-\cos (\vartheta / 2) \sin (\vartheta / 2) & \cos ^{2}(\vartheta / 2) \\
-\sin ^{2}(\vartheta / 2) & \cos (\vartheta / 2) \sin (\vartheta / 2)
\end{array}\right),
$$

where $\vartheta$ is the tilting angle. This is obtained from (2.19) by setting

$$
\alpha=-\eta / 2 \quad e^{-\beta}=\tan (\vartheta / 2) \quad \theta=0 .
$$

Now

$$
\mathcal{N}(0)=-4 \sinh ^{2}(\eta) \cosh ^{2}(\beta)
$$

and for the overlap we get

$$
\frac{\left|\left\langle\Psi(N, \vartheta) \mid\left\{ \pm \lambda^{+}\right\}_{N / 2}\right\rangle\right|^{2}}{\left\langle\left\{ \pm \lambda^{+}\right\}_{N / 2} \mid\left\{ \pm \lambda^{+}\right\}_{N / 2}\right\rangle}=\frac{1}{(2 \cosh (\beta))^{L}} \prod_{j=1}^{N / 2} u_{N, \vartheta}\left(\lambda_{j}\right) \times \frac{\operatorname{det}_{N / 2} G_{j k}^{+}}{\operatorname{det}_{N / 2} G_{j k}^{-}}
$$

with

$$
u_{N, \vartheta}(\lambda)=\frac{v_{\eta / 2}^{s}\left(v_{\beta}^{c}\right)^{2}}{v_{\eta / 2}^{c} v_{0}^{s} v_{0}^{c}}
$$

It can be seen immediately that in the $\beta \rightarrow \infty$ limit the overlaps with $N=L / 2$ converge to those of the original Néel state, whereas for $N<L / 2$ they scale to zero.

\section{Conclusions}

In this work we conjectured the formula (3.13) for exact overlaps with arbitrary two-site states. The conjecture was confirmed by numerical tests, and we have shown that it produces the correct Quench Action TBA in the thermodynamic limit.

It is quite intriguing that the new exact formula involves the same ratio of two Gaudinlike determinants, that were derived originally for the Néel state in 11. For the generalized dimer states given by (2.21) this can be understood through the relation (2.23) which relates the overlaps with them directly to the Néel case. On the other hand, in the generic case we do not know of such a simple relation which would follow immediately from the coordinate Bethe Ansatz wave function.

It might be that for generic two-site states it is only the on-shell case where such a simple final formula can be derived. The result of [34 point towards this possibility: for 
states corresponding to the special case of lower diagonal $K$-matrices the off-shell formula differs from that of the Néel state, but the on-shell case already takes the same form. The recursion relations derived in $[55$ also confirm this scenario, because they show that the generic off-shell case has to have different structure from the original off-shell formula for Néel (2.24).

In the present work we have shown that the Gaudin-like determinants play a special role in the overlap sum rule 2.31) in the thermodynamic limit: their ratio tends to a finite number which cancels two other $\mathcal{O}(1)$ contributions to the Quench Action. These additional contributions come from the modified density of states due to the pair structure, and from the fluctuations around the saddle point solution, in complete analogy with the $\mathcal{O}(1)$ terms in the free energy of a boundary system [39, 40]. The cancellation in the $L \rightarrow \infty$ limit is necessary for the consistency of the Quench Action method, but it is not enough to prove the finite volume formulas. It is worth noting again, that the same determinants already appeared in a specific overlap in the Lieb-Liniger model [61, 12, 13], and analogous determinants were found for overlaps in the $S U(3)$-symmetric model too [27]. Thus they might be generally present in overlaps with integrable initial states.

Future tasks include the construction of a proof of our conjectured formulas, including the result (3.17) for one-site states in chains with odd length. We stress again that the existing methods in the literature were based on the Boundary Algebraic Bethe Ansatz, and this only applies to even volumes. Coordinate Bethe Ansatz calculations don't distinguish between the odd and even cases, thus they could confirm (3.17) for low particle numbers. Alternatively, the recursion relations of [55] could be adapted to the odd length case to find a general off-shell formula.

Also, it would be desirable to develop methods for overlaps in other models. In this work we have argued that factorized overlaps can be found only for integrable initial states. Therefore, in each model the first task is to characterize the integrable states (by integrable $K$-matrices or other tools), such that the overlap calculations can be carried out. An interesting example would be the spin-1 XXZ chain, where the integrable states are given in [28], but the exact overlaps are not yet known. An other example is the $S U(3)$-symmetric chain: the results of [27] for an integrable MPS are obtained from coordinate Bethe Ansatz for small particle numbers, and it is desirable to find a general proof in this case too.

We hope to return to these questions in future research.

\section{Acknowledgments}

We would like to thank Márton Mestyán, Yunfeng Jiang, Lorenzo Piroli, and Eric Vernier for inspiring discussions, and we acknowledge support from the "Premium" Postdoctoral Program of the Hungarian Academy of Sciences, the K2016 grant no. 119204 and the KH17 grant no. 125567 of the research agency NKFIH. This work was partially supported also within the Quantum Technology National Excellence Program (Project No. 2017-1.2.1-NKP2017-00001).

\section{A Numerical tests}

We numerically tested the conjectured general formula (3.13) on finite chains of up to $L=12$ for various sets of parameters $(\alpha, \beta, \gamma)$. The methods and computer programs were the same as those described in [62]: the Bethe states were obtained by diagonalizing transfer matrices combined with $S_{z}$, the transfer matrix eigenvalues were computed numerically for a couple of rapidity parameters, and the so-called $Q$-polynomial was reconstructed using the $T-Q$ relations, which provides and efficient way to determine the Bethe roots. The overlaps were computed by the real space scalar products, and compared to the prediction (3.13).

Numerical data for the overlap with the tilted ferromagnetic state $|X F\rangle \equiv|\Psi(F, \pi / 2)\rangle$ $(\theta=0)$ are listed in Tables $\mathrm{A} 5$. The data correspond to $\Delta=2$ and $L=6,7,8,9,12$. In the tables we list the energy eigenvalues, the number of particles, the set of positive rapidities, 


\begin{tabular}{|c|c|l|c|}
\hline$E$ & $N$ & $\left\{\lambda^{+}\right\}_{N / 2}$ & $\left|\left\langle X F \mid\{ \pm \lambda\}_{N / 2}\right\rangle\right|$ \\
\hline-22.246211 & 2 & $\{0.199867\}$ & 0.017516245 \\
\hline-12.000000 & 2 & $\{0.785398\}$ & 0.153093109 \\
\hline-5.753789 & 2 & $\left\{\frac{\pi}{2}+0.705289 \mathrm{i}\right\}$ & 0.458945183 \\
\hline
\end{tabular}

Table 1: Numerical data for the overlaps with the tilted ferromagnetic state. Here $\Delta=2$ and $L=6$.

\begin{tabular}{|c|c|l|c|}
\hline$E$ & $N$ & $\left\{\lambda^{+}\right\}_{N / 2}$ & $\left|\left\langle X F \mid\{ \pm \lambda\}_{N / 2}\right\rangle\right|$ \\
\hline-22.604374 & 4 & $\left\{\frac{\pi}{2}+0.900409 \mathrm{i}, 0.425131\right\}$ & 0.054097899 \\
\hline-19.517541 & 2 & $\{0.345713\}$ & 0.023806863 \\
\hline-10.904438 & 4 & $\left\{\frac{\pi}{2}+1.620317 \mathrm{i}, 1.098201\right\}$ & 0.287746749 \\
\hline-10.610815 & 2 & $\{0.917864\}$ & 0.160905125 \\
\hline-6.491189 & 4 & $\left\{\frac{\pi}{2}+0.668574 \mathrm{i}, \frac{\pi}{2}+2.216935 \mathrm{i}\right\}$ & 0.433258267 \\
\hline-5.871644 & 2 & $\left\{\frac{\pi}{2}+0.683016 \mathrm{i}\right\}$ & 0.370951849 \\
\hline
\end{tabular}

Table 2: Numerical data for the overlaps with the tilted ferromagnetic state. Here $\Delta=2$ and $L=7$.

and the normalized overlap as obtained from exact diagonalization (corresponding to the square root of (3.17)). We omitted all states that included the singular rapidities $0, \pi / 2$ or exact 2-strings of the form $\lambda \pm i \eta / 2$ with some $\lambda \in \mathbb{R}$; in these cases regularized formulas are needed, which are not discussed in the present work.

The difference between the prediction and the numerical data for the overlap was always smaller than $10^{-12}$, therefore we do not list the errors (at length $L=12$ there were two states with an error of $\mathcal{O}\left(10^{-5}\right)$, but they had 2-strings very close to an exact two-string, and this was identified as the source of the bigger error). We found similar agreement for other $(\alpha, \beta, \theta)$ parameters of the $K$-matrices. We also investigated the $\Delta<1$ regime and found that the overlap formulas are correct for all regular Bethe states (there are a number of singular cases for $\Delta<1$ [42], but these are not discussed here).

\section{References}

[1] V. Korepin, N. Bogoliubov, and A. Izergin, Quantum inverse scattering method and correlation functions. Cambridge University Press, 1993.

[2] B. Sutherland, Beautiful Models. World Scientific Publishing Company, 2004.

[3] M. Takahashi, Thermodynamics of One-Dimensional Solvable Models. Cambridge University Press, 1999.

[4] J. Sato, B. Aufgebauer, H. Boos, F. Göhmann, A. Klümper, M. Takahashi, and C. Trippe, "Computation of Static Heisenberg-Chain Correlators: Control over Length and Temperature Dependence," Physical Review Letters 106 (2011) no. 25, 257201. arXiv:1105.4447 [cond-mat.str-el]

[5] K. K. Kozlowski, "Asymptotic analysis and quantum integrable models," ArXiv e-prints (2015), arXiv:1508.06085 [math-ph].

[6] M. Rigol, V. Dunjko, V. Yurovsky, and M. Olshanii, "Relaxation in a Completely Integrable Many-Body Quantum System: An Ab Initio Study of the Dynamics of the Highly Excited States of 1D Lattice Hard-Core Bosons," Physical Review Letters 98 (2007) no. 5, 050405, arXiv: cond-mat/0604476.

[7] F. H. L. Essler and M. Fagotti, "Quench dynamics and relaxation in isolated integrable quantum spin chains," Journal of Statistical Mechanics: Theory and Experiment 6 (2016) 064002, arXiv:1603.06452 [cond-mat.quant-gas]. 


\begin{tabular}{|c|c|l|c|}
\hline$E$ & $N$ & $\left\{\lambda^{+}\right\}_{N / 2}$ & $\left|\left\langle X F \mid\{ \pm \lambda\}_{N / 2}\right\rangle\right|$ \\
\hline-36.157715 & 4 & $\{0.676922,0.169306\}$ & 0.005996275 \\
\hline-28.296911 & 4 & $\left\{\frac{\pi}{2}+0.762821 \mathrm{i}, 0.159158\right\}$ & 0.017584753 \\
\hline-23.126614 & 2 & $\{0.137851\}$ & 0.005629069 \\
\hline-20.284425 & 4 & $\left\{\frac{\pi}{2}+0.785277 \mathrm{i}, 0.581896\right\}$ & 0.086678331 \\
\hline-19.368479 & 4 & $\{0.041678,1.336520 \mathrm{i}\}$ & 0.000330915 \\
\hline-17.149343 & 2 & $\{0.463317\}$ & 0.025486372 \\
\hline-14.529278 & 4 & $\{0.564749+0.660013 \mathrm{i}, 0.564749-0.660013 \mathrm{i}\}$ & 0.041199177 \\
\hline-10.614015 & 4 & $\left\{\frac{\pi}{2}+1.447586 \mathrm{i}, 1.285445\right\}$ & 0.370979758 \\
\hline-9.790362 & 2 & $\{1.019560\}$ & 0.155431765 \\
\hline-6.749178 & 4 & $\left\{\frac{\pi}{2}+2.041251 \mathrm{i}, \frac{\pi}{2}+0.660208 \mathrm{i}\right\}$ & 0.355324817 \\
\hline-5.933681 & 2 & $\left\{\frac{\pi}{2}+0.671197 \mathrm{i}\right\}$ & 0.290748560 \\
\hline
\end{tabular}

Table 3: Numerical data for the overlaps with the tilted ferromagnetic state. Here $\Delta=2$ and $L=8$.

\begin{tabular}{|c|c|l|c|}
\hline$E$ & $N$ & $\left\{\lambda^{+}\right\}_{N / 2}$ & $\left|\left\langle X F \mid\{ \pm \lambda\}_{N / 2}\right\rangle\right|$ \\
\hline-32.137267 & 4 & $\{0.297161,0.815441\}$ & 0.012313623 \\
\hline-26.460311 & 4 & $\left\{\frac{\pi}{2}+0.714053 \mathrm{i}, 0.283467\right\}$ & 0.029323961 \\
\hline-21.449359 & 2 & $\{0.246322\}$ & 0.007529756 \\
\hline-18.515293 & 4 & $\left\{\frac{\pi}{2}+0.730070 \mathrm{i}, 0.715849\right\}$ & 0.112794815 \\
\hline-15.303138 & 2 & $\{0.562213\}$ & 0.024735464 \\
\hline-13.475088 & 4 & $\{0.816328+0.663186 \mathrm{i}, 0.816328-0.663186 \mathrm{i}\}$ & 0.079753699 \\
\hline-10.585428 & 4 & $\left\{\frac{\pi}{2}+1.377907 \mathrm{i}, 1.397985\right\}$ & 0.389151297 \\
\hline-9.281427 & 2 & $\{1.098337\}$ & 0.141208039 \\
\hline-6.826613 & 4 & $\left\{\frac{\pi}{2}+1.994065 \mathrm{i}, \frac{\pi}{2}+0.658742 \mathrm{i}\right\}$ & 0.273057092 \\
\hline-5.966075 & 2 & $\left\{\frac{\pi}{2}+0.664996 \mathrm{i}\right\}$ & 0.222944498 \\
\hline
\end{tabular}

Table 4: Numerical data for the overlaps with the tilted ferromagnetic state. Here $\Delta=2$ and $L=9$. 


\begin{tabular}{|c|c|c|c|}
\hline$E$ & $N$ & $\left\{\lambda^{+}\right\}_{N / 2}$ & $\left\langle X F \mid\{ \pm \lambda\}_{N / 2}\right\rangle \mid$ \\
\hline-53.840470 & 6 & $\{0.841017,0.368953,0.110942\}$ & 0.000420653 \\
\hline-48.461935 & 6 & $\{\pi / 2+0.724032 \mathrm{i}, 0.354057,0.107515\}$ & 0.000815421 \\
\hline-43.743023 & 4 & $\{0.312266,0.097459\}$ & 0.000213745 \\
\hline-41.474319 & 6 & $\{\pi / 2+0.744061 \mathrm{i}, 0.749083,0.105807\}$ & 0.002427526 \\
\hline-41.396687 & 6 & $\{0.229201,0.049088,1.362248 \mathrm{i}\}$ & 0.000021161 \\
\hline-38.224308 & 4 & $\{0.599580,0.096548\}$ & 0.000583217 \\
\hline-37.591463 & 6 & $\{0.669408+0.659265 \mathrm{i}, 0.109511,0.669408-0.659265 \mathrm{i}\}$ & 0.001058077 \\
\hline-37.576158 & 6 & $\{\pi / 2+0.749424 \mathrm{i}, 0.345238,0.742919\}$ & 0.008821628 \\
\hline-35.944660 & 6 & $\{0.489429,0.024984,1.327009 \mathrm{i}\}$ & 0.000014369 \\
\hline-34.985584 & 4 & $\{0.307780,0.596867\}$ & 0.002112300 \\
\hline-34.043431 & 6 & $\{\pi / 2+1.428778 \mathrm{i}, 1.344761,0.099967\}$ & 0.006989255 \\
\hline-33.462316 & 6 & $\{0.617460+0.658894 \mathrm{i}, 0.366883,0.617460-0.658894 \mathrm{i}\}$ & 0.003462560 \\
\hline-32.822751 & 4 & $\{1.104307,0.094996\}$ & 0.002968724 \\
\hline-30.518925 & 6 & $\{\pi / 2+1.436814 \mathrm{i}, 1.335700,0.321123\}$ & 0.025203737 \\
\hline-30.341773 & 6 & $\{\pi / 2+2.029253 \mathrm{i}, \pi / 2+0.659301 \mathrm{i}, 0.095578\}$ & 0.005249464 \\
\hline-29.657104 & 4 & $\{0.302153,1.100640\}$ & 0.010623914 \\
\hline-29.551789 & 4 & $\{\pi / 2+0.665839 \mathrm{i}, 0.093343\}$ & 0.004360074 \\
\hline-29.325916 & 6 & $\{1.320224 \mathrm{i}, 0.979205,0.014355\}$ & 0.000023370 \\
\hline-27.116875 & 6 & $\{\pi / 2+2.032170 \mathrm{i}, \pi / 2+0.659386 \mathrm{i}, 0.304112\}$ & 0.018973378 \\
\hline-26.470339 & 4 & $\{\pi / 2+0.666183 \mathrm{i}, 0.295965\}$ & 0.015545995 \\
\hline-26.118545 & 6 & $\{0.439354+0.658493 \mathrm{i}, 0.873829,0.439354-0.658493 \mathrm{i}\}$ & 0.007751711 \\
\hline-25.081390 & 6 & $\{1.318468 \mathrm{i}, \pi / 2+0.684113 \mathrm{i}, 0.009694\}$, & 0.000018314 \\
\hline-24.646110 & 6 & $\{\pi / 2+1.468921 \mathrm{i}, 0.630204,1.303662\}$ & 0.066154118 \\
\hline-24.385478 & 4 & $\{0.576317,1.090376\}$ & 0.027193274 \\
\hline-23.655384 & 2 & $\{0.085451\}$ & 0.000827829 \\
\hline-21.746288 & 6 & $\{\pi / 2+2.041388 \mathrm{i}, \pi / 2+0.659683 \mathrm{i}, 0.579956\}$ & 0.049754532 \\
\hline-21.303110 & 4 & $\{\pi / 2+0.667265 \mathrm{i}, 0.559619\}$ & 0.039052708 \\
\hline-21.194242 & 6 & $\{\pi / 2+0.700168 \mathrm{i}, 0.344700+0.658480 \mathrm{i}, 0.344700-0.658480 \mathrm{i}\}$ & 0.010137832 \\
\hline-21.064516 & 2 & $\{0.267132\}$ & 0.002871132 \\
\hline-19.200544 & 4 & $\{0.001717,1.317009 \mathrm{i}\}$ & 0.000000235 \\
\hline-18.964761 & 6 & $\{1.316961 \mathrm{i}, 0.000448,2.668795 \mathrm{i}\}$ & 0.000000015 \\
\hline-17.389558 & 6 & $\{\pi / 2+1.108583 \mathrm{i}, 1.342005+0.507935 \mathrm{i}, 1.342005-0.507935 \mathrm{i}\}$ & 0.103228483 \\
\hline-16.710970 & 2 & $\{0.485898\}$ & 0.006621650 \\
\hline-16.200689 & 6 & $\{\pi / 2+2.074803 \mathrm{i}, \pi / 2+0.661189 \mathrm{i}, 1.062080\}$ & 0.215163928 \\
\hline-15.766469 & 4 & $\{\pi / 2+0.671617 \mathrm{i}, 1.013347\}$ & 0.141856281 \\
\hline-15.756503 & 4 & $\{0.217016+0.658479 \mathrm{i}, 0.217016-0.658479 \mathrm{i}\}$ & 0.001557160 \\
\hline-14.236805 & 4 & $\{0.655902+0.658434 \mathrm{i}, 0.655902-0.658434 \mathrm{i}\}$ & 0.012046104 \\
\hline-13.951915 & 6 & $\{0.700809+1.315956 \mathrm{i}, 0.700110,0.700809-1.315956 \mathrm{i}\}$ & 0.022711987 \\
\hline-12.738629 & 6 & $\{\pi / 2+2.087851 \mathrm{i}, 1.384487+0.663893 \mathrm{i}, 1.384487-0.663893 \mathrm{i}\}$ & 0.254960253 \\
\hline-12.385621 & 4 & $\{1.185112+0.662562 \mathrm{i}, 1.185112-0.662562 \mathrm{i}\}$ & 0.132292301 \\
\hline-12.000000 & 2 & $\{0.785398\}$ & 0.017116330 \\
\hline-10.883883 & 6 & $\{\pi / 2+2.686707 \mathrm{i}, \pi / 2+1.317465 \mathrm{i}, 1.559164\}$ & 0.286298034 \\
\hline-10.654120 & 4 & $\{\pi / 2+1.321734 \mathrm{i}, 1.534546\}$ & 0.260335581 \\
\hline-8.573495 & 2 & $\{1.248484\}$ & 0.084054304 \\
\hline-6.856453 & 4 & $\{\pi / 2+1.975868 \mathrm{i}, \pi / 2+0.658480 \mathrm{i}\}$ & 0.112477548 \\
\hline-5.995635 & 2 & $\{\pi / 2+0.659319 \mathrm{i}\}$ & 0.093286717 \\
\hline
\end{tabular}

Table 5: Numerical data for the overlaps with the tilted ferromagnetic state. Here $\Delta=2$ and $L=12$. 
[8] L. Vidmar and M. Rigol, "Generalized Gibbs ensemble in integrable lattice models," Journal of Statistical Mechanics: Theory and Experiment 6 (2016) 064007 , arXiv: 1604.03990 [cond-mat.stat-mech].

[9] J.-S. Caux and F. H. L. Essler, "Time Evolution of Local Observables After Quenching to an Integrable Model," Physical Review Letters 110 (2013) no. 25, 257203, arXiv:1301.3806 [cond-mat.stat-mech]

[10] B. Pozsgay, "Overlaps between eigenstates of the XXZ spin- $1 / 2$ chain and a class of simple product states," J. Stat. Mech. 2014 (2014) no. 6, P06011, arXiv:1309.4593 [cond-mat.stat-mech].

[11] M. Brockmann, J. De Nardis, B. Wouters, and J.-S. Caux, "A Gaudin-like determinant for overlaps of Néel and XXZ Bethe states," Journal of Physics A Mathematical General 47 (2014) no. 14, 145003, arXiv: 1401.2877 [cond-mat.stat-mech].

[12] M. Brockmann, J. De Nardis, B. Wouters, and J.-S. Caux, "Néel-XXZ state overlaps: odd particle numbers and Lieb-Liniger scaling limit," Journal of Physics A Mathematical General 47 (2014) 345003, arXiv:1403.7469 [cond-mat.stat-mech].

[13] M. Brockmann, "Overlaps of q-raised Néel states with XXZ Bethe states and their relation to the Lieb-Liniger Bose gas," Journal of Statistical Mechanics: Theory and Experiment 5 (2014) 05006, arXiv:1402.1471 [cond-mat.stat-mech].

[14] B. Wouters, J. De Nardis, M. Brockmann, D. Fioretto, M. Rigol, and J.-S. Caux, "Quenching the Anisotropic Heisenberg Chain: Exact Solution and Generalized Gibbs Ensemble Predictions," Physical Review Letters 113 (2014) no. 11, 117202, arXiv:1405.0172 [cond-mat.str-el].

[15] B. Pozsgay, M. Mestyán, M. A. Werner, M. Kormos, G. Zaránd, and G. Takács, "Correlations after Quantum Quenches in the XXZ Spin Chain: Failure of the Generalized Gibbs Ensemble," Physical Review Letters 113 (2014) no. 11, 117203, arXiv:1405.2843 [cond-mat.stat-mech]

[16] E. Ilievski, M. Medenjak, T. Prosen, and L. Zadnik, "Quasilocal charges in integrable lattice systems," Journal of Statistical Mechanics: Theory and Experiment 6 (2016) 064008, arXiv: 1603.00440 [cond-mat.stat-mech].

[17] E. Ilievski, J. De Nardis, B. Wouters, J.-S. Caux, F. H. L. Essler, and T. Prosen, "Complete Generalized Gibbs Ensembles in an Interacting Theory," Physical Revieu Letters 115 (2015) no. 15, 157201, arXiv:1507.02993 [quant-ph].

[18] B. Doyon, "Thermalization and pseudolocality in extended quantum systems," Communications in Mathematical Physics 351 (2017) no. 1, 155-200. arXiv:1512.03713 [math-ph].

[19] E. Ilievski, E. Quinn, and J.-S. Caux, "From interacting particles to equilibrium statistical ensembles," Phys. Rev. B 95 (2017) no. 11, 115128, arXiv:1610.06911 [cond-mat.stat-mech].

[20] B. Pozsgay, E. Vernier, and M. Werner, "On Generalized Gibbs Ensembles with an infinite set of conserved charges," Journal of Statistical Mechanics: Theory and Experiment 9 (2017) 093103, arXiv:1703.09516 [cond-mat.stat-mech].

[21] E. Ilievski, E. Quinn, J. De Nardis, and M. Brockmann, "String-charge duality in integrable lattice models," Journal of Statistical Mechanics: Theory and Experiment 6 (2016) 063101, arXiv:1512.04454 [cond-mat.stat-mech].

[22] L. Piroli, E. Vernier, and P. Calabrese, "Exact steady states for quantum quenches in integrable Heisenberg spin chains," Physical Review B 94 (2016) no. 5, 054313, arXiv:1606.00383 [cond-mat.stat-mech].

[23] V. Alba and P. Calabrese, "The quench action approach in finite integrable spin chains," Journal of Statistical Mechanics: Theory and Experiment 4 (2016) 043105 , arXiv: 1512.02213 [cond-mat.str-el]. 
[24] V. Alba and P. Calabrese, "Quench action and Rényi entropies in integrable systems," Phys Rev B 96 (2017) no. 11, 115421, arXiv:1705.10765 [cond-mat.stat-mech].

[25] V. Alba and P. Calabrese, "Rényi entropies after releasing the Néel state in the XXZ spin-chain," Journal of Statistical Mechanics: Theory and Experiment 11 (2017) 113105, arXiv:1709.02193 [cond-mat.stat-mech].

[26] M. Mestyán, B. Bertini, L. Piroli, and P. Calabrese, "Exact solution for the quench dynamics of a nested integrable system," Journal of Statistical Mechanics: Theory and Experiment 8 (2017) 083103, arXiv:1705.00851 [cond-mat.stat-mech]].

[27] M. de Leeuw, C. Kristjansen, and S. Mori, "AdS/dCFT one-point functions of the SU(3) sector," Physics Letters B 763 (2016) 197-202, arXiv:1607.03123 [hep-th].

[28] L. Piroli, B. Pozsgay, and E. Vernier, "What is an integrable quench?," Nuclean Physics B 925 (2017) no. Supplement C, 362-402, arXiv:1709.04796 [cond-mat.stat-mech].

[29] S. Sotiriadis, G. Takacs, and G. Mussardo, "Boundary state in an integrable quantum field theory out of equilibrium," Physics Letters B 734 (2014) 52-57, arXiv: 1311.4418 [cond-mat.stat-mech].

[30] D. X. Horváth, S. Sotiriadis, and G. Takács, "Initial states in integrable quantum field theory quenches from an integral equation hierarchy," Nuclear Physics B 902 (2016) 508-547, arXiv:1510.01735.

[31] D. X. Horváth and G. Takács, "Overlaps after quantum quenches in the sine-Gordon model," Physics Letters B 771 (2017) 539-545, arXiv:1704.00594 [cond-mat.stat-mech].

[32] M. de Leeuw, C. Kristjansen, and K. Zarembo, "One-point functions in defect CFT and integrability," Journal of High Energy Physics 8 (2015) 98, arXiv:1506.06958 [hep-th].

[33] I. Buhl-Mortensen, M. de Leeuw, C. Kristjansen, and K. Zarembo, "One-point Functions in AdS/dCFT from Matrix Product States," Journal of High Energy Physics 2 (2016) 52, arXiv:1512.02532 [hep-th].

[34] O. Foda and K. Zarembo, "Overlaps of Partial Neel States and Bethe States," Journa of Statistical Mechanics: Theory and Experiment 2016 (2015) no. 2, 023107, arXiv:1512.02533 [hep-th].

[35] K. K. Kozlowski and B. Pozsgay, "Surface free energy of the open XXZ spin-1/2 chain," J. Stat. Mech. 2012 (2012) no. 05, 21.

[36] F. Göhmann, M. Bortz, and H. Frahm, "Surface free energy for systems with integrable boundary conditions," Journal of Physics A Mathematical General 38 (2005) 10879-10891, arXiv: cond-mat/0508377.

[37] P. Dorey, I. Runkel, R. Tateo, and G. Watts, "g-function flow in perturbed boundary conformal field theories," Nucl. Phys. B578 (2000) 85-122, arXiv:hep-th/9909216.

[38] P. Dorey, C. Rim, and R. Tateo, "Exact g-function flow between conformal field theories," Nuclear Physics B 834 (2010) 485-501, arXiv:0911.4969 [hep-th].

[39] B. Pozsgay, "On O(1) contributions to the free energy in Bethe Ansatz systems: the exact g-function," JHEP 08 (2010) 090, arXiv:1003.5542 [hep-th].

[40] F. Woynarovich, "On the normalization of the partition function of Bethe Ansatz systems," Nuclear Physics B 852 (2010) 269-286, arXiv:1007.1148 [cond-mat.str-el]].

[41] L. Piroli, B. Pozsgay, and E. Vernier, "From the Quantum Transfer Matrix to the Quench Action: The Loschmidt echo in XXZ Heisenberg spin chains," Journal of Statistical Mechanics: Theory and Experiment 2017 (2017) no. 2, 023106, arXiv:1611.06126 [cond-mat.stat-mech].

[42] R. J. Baxter, "Completeness of the Bethe ansatz for the six and eight-vertex models," Journal of Statistical Physics 108 (2002) no. 1, 1-48, cond-mat/0111188. 
[43] H. Bethe, "Zur Theorie der Metalle," Zeitschrift für Physik A71 (1931) 205.

[44] R. Orbach, "Linear Antiferromagnetic Chain with Anisotropic Coupling," Physica Review 112 (1958) no. 2, 309-316.

[45] L. R. Walker, "Antiferromagnetic Linear Chain," Physical Review 116 (1959) no. 5, 1089-1090.

[46] C. N. Yang and C. P. Yang, "One-Dimensional Chain of Anisotropic Spin-Spin Interactions. I. Proof of Bethe's Hypothesis for Ground State in a Finite System," Physical Review 150 (1966) no. 1, 321-327.

[47] M. Grabowski and P. Mathieu, "Structure of the conservation laws in integrable spin chains with short range interactions," Annals Phys. 243 (1995) 299-371, arXiv:hep-th/9411045 [hep-th].

[48] I. V. Cherednik, "Factorizing Particles on a Half Line and Root Systems," Theor. Math. Phys. 61 (1984) 977-983.

[49] E. K. Sklyanin, "Boundary conditions for integrable quantum systems," J. Phys. A 21 (1988) no. 10, 2375 .

[50] H. J. de Vega and A. Gonzalez Ruiz, "Boundary K-matrices for the six vertex and the $\mathrm{n}(2 \mathrm{n}-1) \mathrm{A}_{n-1}$ vertex models," Journal of Physics A Mathematical General 26 (1993) L519-L524, hep-th/9211114.

[51] M. Mestyán, B. Pozsgay, G. Takács, and M. A. Werner, "Quenching the XXZ spin chain: quench action approach versus generalized Gibbs ensemble," Journal of Statistical Mechanics: Theory and Experiment 4 (2015) 1, arXiv: 1412.4787 [cond-mat.stat-mech].

[52] O. Tsuchiya, "Determinant formula for the six-vertex model with reflecting end," Journal of Mathematical Physics 39 (1998) no. 11, 5946-5951, arXiv:9804010 [solv-int].

[53] V. E. Korepin, "Calculation of norms of Bethe wave functions," Comm. Math. Phys. 86 (1982) 391.

[54] A. G. Izergin and V. E. Korepin, "Correlation functions for the Heisenberg XXZ-antiferromagnet," Comm. Math. Phys. 99 (1985) 271.

[55] L. Piroli and P. Calabrese, "Recursive formulas for the overlaps between Bethe states and product states in XXZ Heisenberg chains," Journal of Physics A: Mathematical and Theoretical 47 (2014) no. 38, 385003, arXiv:1407.2242 [cond-mat.stat-mech].

[56] A. N. Kirillov and V. E. Korepin, "Norms of bound states," Journal of Soviet Mathematics 40 (1988) no. 1, 13-21.

[57] V. E. Korepin, "Correlation functions of the one-dimensional Bose gas in the repulsive case," Comm. Math. Phys. 94 (1984) 93.

[58] C. Destri and J. H. Lowenstein, "Normalization of Bethe-ansatz states in the chiral-invariant Gross-Neveu model," Journal of Mathematical Physics 24 (1983) no. 3, 677-682.

[59] F. Woynarovich, "O(1) contribution of saddle point fluctuations to the free energy of Bethe Ansatz systems," Nucl. Phys. B700 (2004) 331, arXiv: cond-mat/0402129.

[60] B. Pozsgay, "Dynamical free energy and the Loschmidt-echo for a class of quantum quenches in the Heisenberg spin chain," Journal of Statistical Mechanics: Theory and Experiment 2013 (2013) no. 10, P10028, hrXiv:1308.3087 [cond-mat.stat-mech].

[61] J. De Nardis, B. Wouters, M. Brockmann, and J.-S. Caux, "Solution for an interaction quench in the Lieb-Liniger Bose gas," Physical Review A 89 (2014) 033601, arXiv:1308.4310 [cond-mat.stat-mech].

[62] M. Mestyán and B. Pozsgay, "Short distance correlators in the XXZ spin chain for arbitrary string distributions," Journal of Statistical Mechanics: Theory and Experiment 9 (2014) 20, arXiv:1405.0232 [cond-mat.stat-mech]]. 